\title{
Preparation and Characterization of Liposomal Everolimus by Thin-Film Hydration Technique
}

\author{
Gabriela Torres-Flores, ${ }^{1}$ Azucena Gonzalez-Horta, ${ }^{2}$ Yadira I. Vega-Cantu, ${ }^{1}$ \\ Ciro Rodriguez, ${ }^{1,3}$ and Aida Rodriguez-Garcia ${ }^{4}{ }^{4}$ \\ ${ }^{1}$ Tecnologico de Monterrey, Escuela de Ingeniería y Ciencias, Monterrey 64849, Mexico \\ ${ }^{2}$ Universidad Autónoma de Nuevo León, Facultad de Ciencias Biológicas, Laboratorio de Ciencias Genómicas, \\ San Nicolás de los Garza 66455, Mexico \\ ${ }^{3}$ Laboratorio Nacional de Manufactura Aditiva y Digital (MADiT), Apodaca 66629, Mexico \\ ${ }^{4}$ Universidad Autónoma de Nuevo León, Facultad de Ciencias Biológicas, Instituto de Biotecnología, \\ San Nicolás de los Garza 66455, Mexico \\ Correspondence should be addressed to Aida Rodriguez-Garcia; aidrod@hotmail.com
}

Gabriela Torres-Flores and Azucena Gonzalez-Horta contributed equally to this work.

Received 3 July 2020; Revised 25 September 2020; Accepted 28 September 2020; Published 10 October 2020

Academic Editor: Margarita S. Dominguez

Copyright (C) 2020 Gabriela Torres-Flores et al. This is an open access article distributed under the Creative Commons Attribution License, which permits unrestricted use, distribution, and reproduction in any medium, provided the original work is properly cited.

\begin{abstract}
In $10 \%$ to $40 \%$ of the cases of coronary stent implantation, patients face in-stent restenosis due to an inflammatory response, which induces artery thickening. Everolimus, a drug that inhibits growth factor-stimulated cell proliferation of endothelial cells, represents a promising alternative to prevent in-stent restenosis. In this study, everolimus was encapsulated by a film hydration technique in liposomes by using phosphatidylcholine and cholesterol at different ratios. As the ratio of cholesterol increases, it modulates the rigidity of the structure which can affect the encapsulation efficiency of the drug due to steric hindrance. Moreover, various lipid: drug ratios were tested, and it was found that as the lipid: drug ratio increases, the encapsulation efficiency also increases. This behavior is observed because everolimus is a hydrophobic drug; therefore, if the lipidic region increases, more drug can be entrapped into the liposomes. In addition, stability of the encapsulated drug was tested for 4 weeks at $4^{\circ} \mathrm{C}$. Our results demonstrate that it is possible to prepare liposomal everolimus by film hydration technique followed by extrusion with high entrapment efficiency as a viable drug delivery system.
\end{abstract}

\section{Introduction}

Cardiovascular disease remains the leading cause of morbidities and mortality worldwide. It encompasses a broad range of conditions that affect the heart and blood vessels, many of which are related to a process called atherosclerosis. Atherosclerosis is characterized by plaques that appear in the inner layers of arteries. Despite the advances in prevention, controlling risk factors such as smoking, dietary habits, lack of exercise, and high blood pressure, atherosclerosis continues to take deadly toll globally [1]. One of the widely applied percutaneous coronary interventions (PCI) in this type of disease is the coronary stent (coronary intravascular implant) that induces artery revascularization. Despite its popularity, up to 4 out of 10 people with coronary stents present in-stent restenosis, a decrease in the diameter of the arterial lumen [2]. This phenomenon is caused by neointimal proliferation, that is an excessive propagation of the endothelial tissue in the interior area of the stent or by new atherosclerotic plaques [3]. One way to prevent this effect is by using coated stents that allow drug release (such as everolimus, sirolimus, paclitaxel, and zotarolimus) directly into the artery [4]. There are several excipients that can be used for drug release, including polymeric, nanoparticle, and liposomal coatings. 


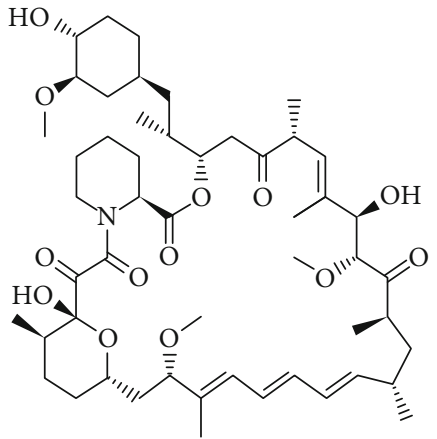

(a)

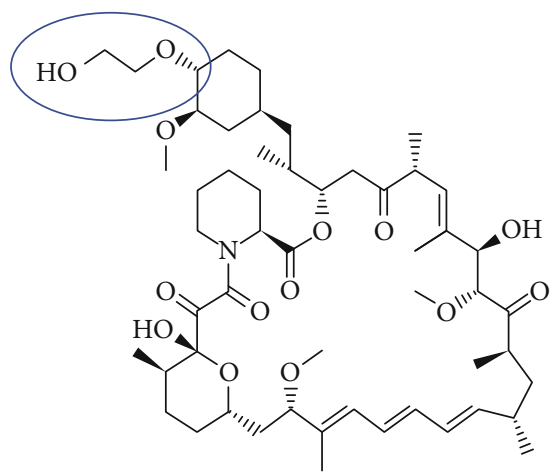

(b)

Figure 1: Drug comparison. Chemical structure of (a) sirolimus and (b) everolimus.

Liposomes are considered as one of the most used nanometric carriers in controlled release systems due to their ability to encapsulate both hydrophilic and hydrophobic drugs [5]. In addition, liposomes are used for drug release systems due to the variety of sizes, biocompatibility, ability to be directed to the site of interest, and their ability to maximize encapsulation capacity by transforming vesicles from multilamellar to unilamellar [6, 7]. Liposomes are composed of either palmitoyl oleyl phosphatidylcholine (POPC) or dioleoyl phosphatidylcholine (DOPC), which belong to the phosphatidylcholine (PC) family, the major component of animal cell membranes [8]. POPC contains one saturated and one unsaturated alkyl chain, while DOPC has two unsaturated alkyl chain; as a result, both have the same phase behaviors at $25^{\circ} \mathrm{C}$. POPC has a phase transition melting temperature $\left(T_{m}\right)$ of $-2^{\circ} \mathrm{C}$ while the $T_{m}$ for DOPC is $-20^{\circ} \mathrm{C}$; thus they are in the liquid crystalline phase. Liposomes can be grafted with polymer coating onto metal-based stents to prolong the half-life of the drugs, by allowing their gradual release and restraining the occurrence of restenosis. Antiproliferative drugs as in the first-generation stents, such as sirolimus and paclitaxel, are widely used to prevent excessive growth of endothelial tissue that could induce in-stent restenosis. However, due to their nature, they delay the process of healing of the artery, leading to a space where platelets agglomerate and cause thrombosis [9]. Due to these side effects, the use of biocompatible polymers in the secondgeneration drug eluting stents may present a higher safety profile, mechanical support, drug-delivery activity, and a complete bioresorption over several years, compared with those of the first generation [10]. Everolimus has been proposed to be used in second-generation coronary stents since, despite its hydrophobic nature, it is more bioavailable than sirolimus. Therefore, it has a greater capacity to be absorbed by the body as the variation in the functional group attached to carbon 40 (circled in Figure 1) increases its polarity [11].

Previously implemented methods for liposome synthesis, specifically for encapsulation of everolimus, solely concentrate on encapsulation by injection [12] and by mixing in aqueous solution [13]. Moreover, these systems and developed methods were mainly targeted at cancer treatment. Unlike previous works, the objective of this study was to develop everolimus-releasing liposomes through thin-film hydration method that could potentially be used to cover coronary stents. This method has presented greater control over size than the reports of the literature mentioned above, which is useful to regulate liberation kinetics as liposomes with smaller sizes $(50 \mathrm{~nm})$ tend to decompose earlier than larger vesicles since their high curvature tension leads to lower stability. Specifically, this work shows the optimization of the molar ratio between PC and cholesterol (Chol), the extent of the double bonds presents in $\mathrm{PC}$, and the lipid: drug ratio for the development of stable unilamellar nanoliposomes using thin-film hydration and extrusion. This fine tuning was aimed at obtaining liposomes between 100 and $150 \mathrm{~nm}$, small enough to avoid an unintended immune response and large enough to maintain liposome stability for at least 4 weeks.

\section{Materials and Methods}

2.1. Materials. Lipids used, cholesterol (Chol), palmitoyl-2oleoyl-sn-glycero-phosphocholine (POPC), and 1,2-dioleoylsn-glycero-3-phosphocholine (DOPC) were purchased from Sigma Aldrich (St. Louis, MO, USA). Everolimus was obtained from Adooq Bioscience (Irvine, CA, USA) and organic solvents from Merck (Massachusetts, USA). On the other hand, HEPES ([4-(2-hydroxyethyl) piperazine-1-ethanesulfonic acid]) buffer and reactive grade sodium chloride were purchased from CTR Scientific S.A de C.V (Monterrey, Mex).

\subsection{Methods}

2.2.1. Preparation of Liposomal Everolimus. In the present work, in order to analyze the effect of drug to lipid ratio, liposomal composition of samples $\mathrm{A}, \mathrm{B}$, and $\mathrm{C}$ was maintained at a 9:1 w/w ratio of palmitoyl oleyl PC and Chol, respectively. To follow an optimization process of one factor at a time (OFAT), the lipid:drug with higher entrapment efficiency (\%EE) was chosen for the optimization of the lipid composition. Therefore, the liposome composition was modified using POPC: Chol with a 7:3 ratio and another formulation with DOPC: Chol with a 9:1 ratio as Table 1 suggests. This table indicates the sample name, if the liposome was 
TABle 1: Experimental design for the optimization of liposomes' lipid composition and lipid:drug ratio (concentration of everolimus was maintained at $0.06 \mathrm{mM}$ ).

\begin{tabular}{lccc}
\hline Sample & Lipidic components & $\begin{array}{c}\text { Lipid ratio } \\
\text { PC: Chol }\end{array}$ & $\begin{array}{c}\text { Lipid: drug } \\
\text { molar ratio }\end{array}$ \\
\hline Blank & POPC: Chol & $9: 1$ & - \\
A & POPC: Chol & $9: 1$ & $60: 1$ \\
B & POPC:Chol & $9: 1$ & $45: 1$ \\
C & POPC: Chol & $9: 1$ & $30: 1$ \\
D & POPC:Chol & $7: 3$ & $60: 1$ \\
E & DOPC:Chol & $9: 1$ & $60: 1$ \\
\hline
\end{tabular}

prepared using POPC or DOPC, the ratio of either POPC or DOPC to cholesterol, and the molar ratio of everolimus versus the lipidic components, in respective columns. For reproducibility purposes, each sample was produced in triplicate.

To carry out the preparation of lipid mixtures with the absence or presence of everolimus, lipids were dissolved in chloroform: methanol in 3:1 v/v, while everolimus was dissolved in methanol. These solutions were mixed at different molar ratios as shown in Table 1, where the concentration of the drug was maintained at $0.06 \mathrm{mM}$ (Table 1). We relied on the reports of the literature in selecting the lipid:drug ratio for sustained high entrapment efficiency of sirolimus, the drug from which everolimus is derived [14].

After solution preparation, the organic solvent was evaporated under nitrogen flow, followed by 2 hours of maintaining the samples under vacuum with a Vacufuge Plus equipment (New York, USA) to completely remove all traces of the solvent. The lipid film was then hydrated in $1 \mathrm{~mL}$ of buffer and $50 \mathrm{mM}$ HEPES containing $30 \mathrm{mM} \mathrm{NaCl} \mathrm{pH} 7$ at $25^{\circ} \mathrm{C}$ with stirring at $1400 \mathrm{rpm}$ for 2 minutes every 10 minutes during two hours with a Thermomixer Comfort (Eppendorf). The multivesicular (MLV) suspension was then extruded 12 times through $100 \mathrm{~nm}$ pore size nucleopore polycarbonate membranes (Whatman, UK) to produce samples with a narrow size distribution. The extrusion was carried out at $25^{\circ} \mathrm{C}$ to maintain vesicles above phase transition temperature using a miniextrusion kit (Avanti Polar Lipids, Alabaster, AL, USA). The entrapment efficiency of everolimus was calculated by separating the nonencapsulated from the encapsulated drugs by gel filtration chromatography using a Sephadex G75 column. During the elution, 60 fractions of $2 \mathrm{~mL}$ each were collected. Under these experimental conditions, the initial lipid sample was diluted 10 times as determined by phosphorous assay $[15,16]$.

2.2.2. Entrapment Efficiency. The entrapment efficiency (\%EE) analysis was performed by UV-Vis spectroscopy Genesys 10 Thermo-Scientific (Madison, WI, USA) at $278 \mathrm{~nm}$ (lmax of everolimus) and interpolating the value on a calibration curve prepared for this purpose $(y=61.859 x+0.0074)$. The calibration curve was linear in the range of $0-0.006 \mathrm{mM}$ with a correlation coefficient of $R^{2}=0.99871$.

The equation used to calculate the \%EE is shown below, where CS is the sample concentration and $0.006 \mathrm{mM}$ is the theoretical concentration of everolimus (which was diluted by a factor of ten due to the purification process). It should be noted that CS was calculated using the maximum absorbance peak from the extraction profiles of each sample.

$$
\% \mathrm{EE}=\frac{\mathrm{CS}}{0.006} * 100
$$

Equation (1) was used to calculate the \%EE of each sample.

2.2.3. Physicochemical Characterization of Liposomes. Size of empty and loaded liposomes and polydispersity of samples, as well as zeta potential were determined using dynamic light scattering (DLS). These parameters were studied during 4 weeks as a stability analysis using the ZetaSizer Nano equipment ZS90 (Malvern Instruments, UK). Liposomes were stored at $4^{\circ} \mathrm{C}$ during the course of these 4 weeks.

For infrared spectroscopy analysis, the samples were completely dried at $30^{\circ} \mathrm{C}$, using an aqueous solvent group configuration with the equipment Ez-2plus (Genevac) which concentrates samples under vacuum conditions. Once dried, the samples were analyzed by ATR-FTIR spectroscopy (infrared spectroscopy by total attenuated reflectance) using a diamond crystal sample holder in a Perkin Elmer equipment Spectrum 400.

2.2.4. Morphological Characterization of Liposomes. For the morphological study of liposomes, samples were centrifuged with $30 \mathrm{~K}$ filters at $13,500 \mathrm{rpm}$ for 15 minutes to eliminate the salts. These filters retain liposomes since they are heavier than $30 \mathrm{KDaltons}$ allowing the separation of salts. Liposomes were then placed in a scanning electron microscope (SEM) sample holder and allowed to dry in a desiccator. Subsequently, the samples were coated with a $5 \mathrm{~nm}$ gold layer using a rotary pumped coater Quorum Q150R ES, and the morphology was observed by a Zeiss EVO MA25 SEM.

2.2.5. Statistical Analysis. Statistical analysis was performed based on an adjustment to an ANOVA of 2 variables through the GraphPad Prism 8.1.0 software. For all comparative tests, the Tukey test was used and the differences of $p<0.05$ were considered statistically significant.

\section{Results and Discussion}

3.1. Characterization of Liposome Size, Polydispersity, and Stability. Liposomes were developed through thin-film hydration technique, which is the most common method for liposome preparation. Since lipidic composition and concentration could have a significant impact on developing a therapeutically efficient liposomal carrier system, we examined different parameters such as lipid-to-drug molar ratio, the increase in cholesterol, and the presence of more unsaturations in phospholipid's acyl chain. Liposomes' sizes were determined through dynamic light scattering (DLS), where it was observed that sizes were slightly greater than $100 \mathrm{~nm}$, even though they were extruded by a polycarbonate filter with $0.1 \mu \mathrm{m}$ pores, as shown in Figure 2(a). This is due to a squeezing phenomenon of liposomes through the filter. Since cholesterol has a rigid ring and an ultra-smooth face in its 


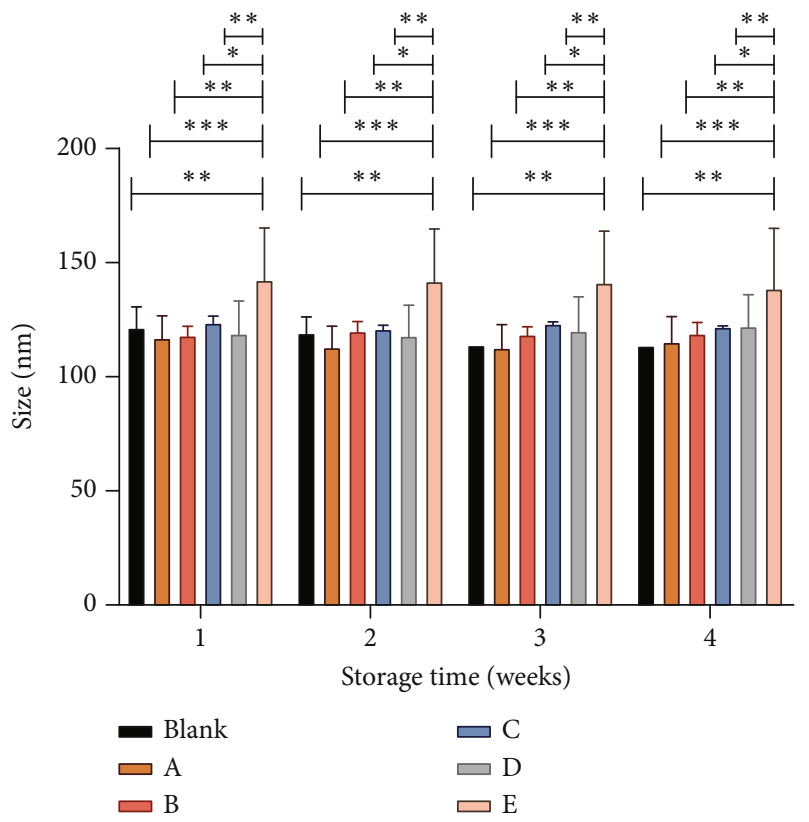

(a)

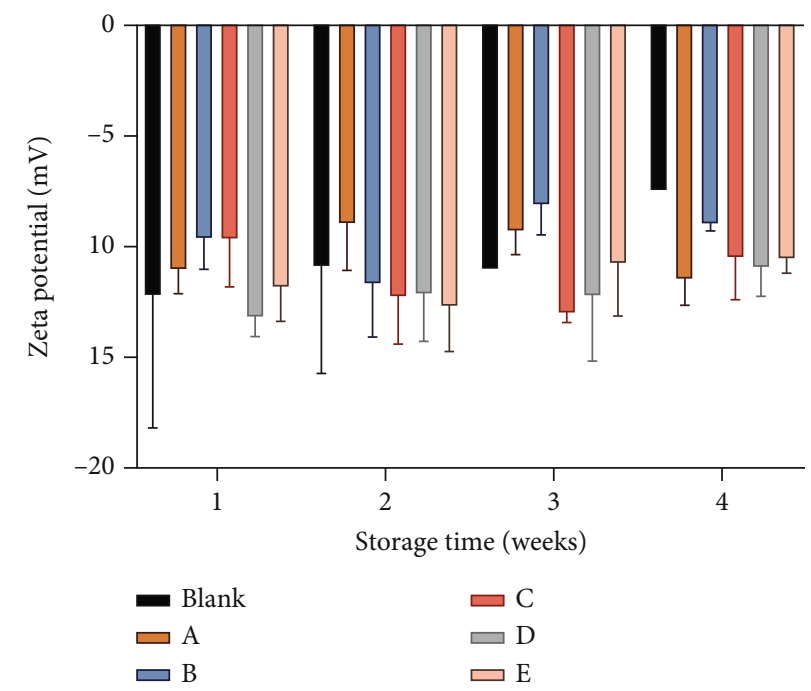

(b)

FiguRe 2: Liposomes' characterization: (a) size and (b) zeta potential. Lines are shown only when there is a significant difference between two samples, in which asterisks denote the level of significance. ${ }^{*} p<0.05,{ }^{* *} p<.001$, and ${ }^{* * *} p<.0001$.

structure, the squeezing process has most likely taken place by allowing the lipids to turn quickly at the extremities [17]. This leads to lipids of high lateral mobility, as well as packed acyl chains [18]. Consequently, this contributes to the variation of sizes since the packing factor affects the intensity and duration of van der Waals forces present in liposomes. In addition, by increasing the percentage of Chol in the formulation, the coexistence of a binary phase Lo (ordered liquid) and Ld (disordered liquid) leads to discontinuities in the lipid membrane, which facilitates the structural interaction between liposomes [19]. This structural interaction propitiates vesicular aggregations which may lead to slightly bigger liposomes. However, the range of sizes obtained was close to the ideal sizes $(100 \mathrm{~nm}-150 \mathrm{~nm})$ for the intended application, as liposomes have to be small enough to "fool" the immune system. However, the liposomes' size should not be below $50 \mathrm{~nm}$ since the stability of liposomes would be compromised due to curvature tension in the vesicle. Moreover, in this specific case, samples with polydispersity index (PDI) less than 0.2 were observed, which indicates that the samples were homogeneous and stable for up to 4 weeks.

It is observed that for sample A (Table 1), the sizes decrease compared to the blank, despite being loaded with everolimus. Loading the liposomes with everolimus did not change the size. This is due to the fact that everolimus forms bonding sites between everolimus and liposomes. Therefore, interaction forces make the liposomes rather compact. Moreover, neither the drug: lipid ratio variation (samples A, B, and C) nor the increment of cholesterol in liposomes (sample D) has shown statistically different size changes (statistical analysis can be reviewed in Supplementary Information (available here)); thus, it can be concluded that these factors do not affect the liposome size. The only sample that showed a significant difference $(p \leq 0.05)$ with respect to the size of the blank was sample E, which was prepared with DOPC instead of POPC. We believe that this is due to the presence of the extra double bond of DOPC which decreases the packing factor of acyl chains; thus, the sizes increase.

On the other hand, Figure 2(b) shows that the zeta potential of all liposomes, primarily composed of PC, was slightly negative $(-11.28 \pm 1.44)$ even though $\mathrm{PC}$ is considered a zwitterion. This is due to a $5 \%$ phosphatidic acid present in POPC and DOPC composition and the negative charge of cholesterol at pH 7 [20]. Furthermore, it was determined that the zeta potential did not show statistically significant changes from weeks 1 to 4 . This indicates that liposomes are stable for the period of 1 month at $4^{\circ} \mathrm{C}$, since a change in zeta potential would suggest a degradation or change in composition [21].

3.2. Chemical Analysis of Liposomes. The ATR-FTIR spectra of the blank (empty liposomes), pure everolimus, and samples A-D (loaded liposomes) are shown in Figure 3. The signal at $1741 \mathrm{~cm}^{-1}$ is attributed to the ester present in everolimus and in the polar head of PC [22]. The band at $1658 \mathrm{~cm}^{-1}$ present in both empty and loaded liposomes is attributed to the $\mathrm{C}=\mathrm{C}$ bond. This last peak has been observed at a wavelength of $1645 \mathrm{~cm}^{-1}$ in polymeric stents coated with everolimus [23]. The peaks at 1658 and $1741 \mathrm{~cm}^{-1}$ are characteristic of the drug. However, the intensity of the previous two signals is lower in loaded liposomes since everolimus is dispersed within the liposome matrix. Moreover, the intensity is low due to the attenuation of ATR spectroscopy signal as a function of the thickness of the diamond crystal used in these assays. The peak at 


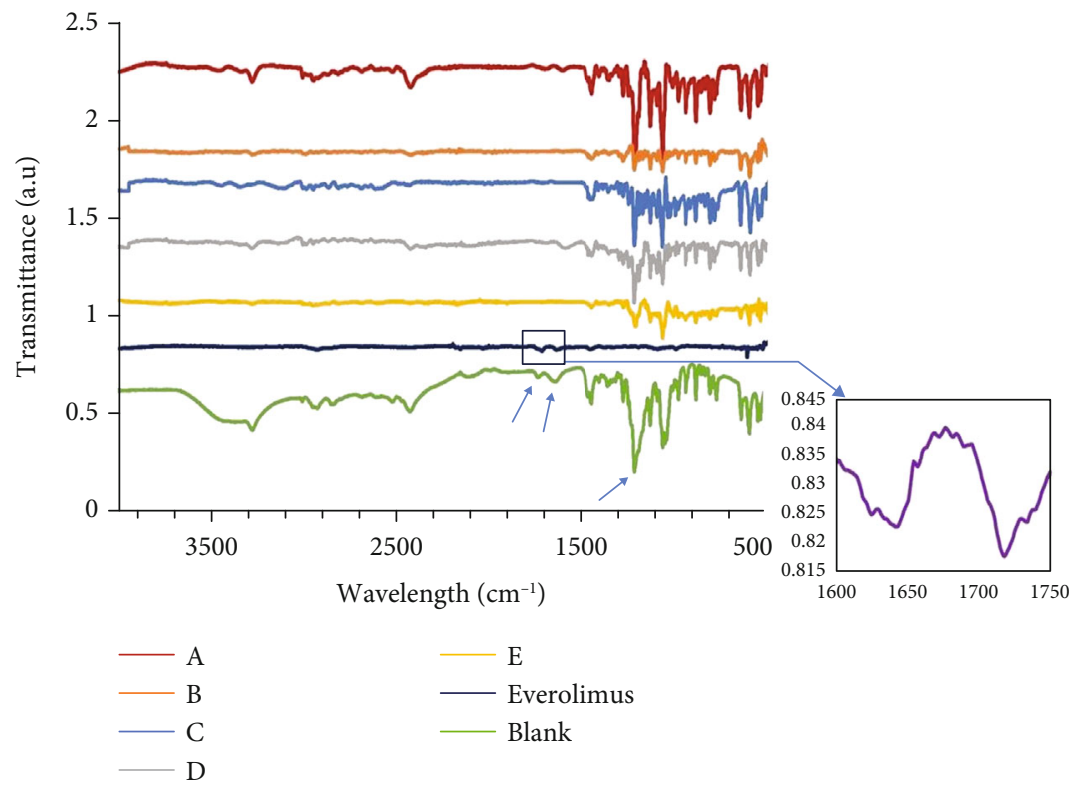

Figure 3: Functional group analysis. ATR-FTIR spectra of samples A-E, free everolimus, and blank liposomes.

$1214 \mathrm{~cm}^{-1}$ in lipid samples represents the C-N bond of PC. It can be observed that sample A shows greater intensity than samples $\mathrm{B}$ and $\mathrm{C}$ as it has the greatest lipid:drug ratio. Similarly, in sample D, POPC decreased; therefore, the peak intensity of this particular sample is less than samples A, B, and $C$. In addition, it was observed that the modification from POPC to DOPC present in sample $\mathrm{E}$ decreased the signal intensity even though the lipid: drug rate was maintained the same. The results prove that there are no new chemical bonds between everolimus and lipids even though they interact with each other at the physical level.

3.3. Entrapment Efficiency. The \%EE analysis was determined with UV-Vis spectroscopy at $278 \mathrm{~nm}$, which corresponds to the active UV functional groups in everolimus. Figure 4 shows the results obtained from samples Figures 4(a)-4(e), where fraction 16 has the maximum absorbance at $278 \mathrm{~nm}$ and $400 \mathrm{~nm}$, which indicate the absorbance of everolimus and the lipids, respectively. Subsequently, it can be observed that approximately 10 fractions are present where the absorbance at $278 \mathrm{~nm}$ rises again, which indicates the presence of free everolimus. This signal appears in fractions collected later compared to the peak of liposomes, as the drug has a lower molecular weight thus taking longer to leave the column which has high area within the pores of the stationary phase.

The $\% \mathrm{EE}$ was calculated against the final concentration of everolimus, which was set to $0.006 \mathrm{mM}$ due to the 10 factor of dilution that was previously stated for the purification method. The blank presented an average \%EE of $11.5 \%$ even though no drug was loaded. This is due to a small variation of the dilution factor during purification. Moreover, the \%EE of samples $A, B$, and $C$, in which the sole difference was the lipid:drug ratio (60:1, 45:1, and $30: 1$, respectively), has a direct relation with the composition. At a higher amount of lipid, the encapsulation efficiency increases. The highest encapsulation percentage was obtained for a lipid:drug
60:1 ratio, since the everolimus is hydrophobic and is trapped within the bilipid layer of liposomes. However, by decreasing the amount of lipid, the number of sites where everolimus can be trapped decreases. This trend is in accordance with a previous study regarding the encapsulation efficiency of another class of hydrophobic drug called docetaxel. In this study conducted by Pereira et al., formulations with 40:1 and 20:1 lipid ratios had encapsulation efficiency of nearly $100 \%$ without significant differences [12]. In that study, however, it was necessary to increase the lipid: drug ratio to $60: 1$ to reach the encapsulation efficiencies close to $100 \%$ since everolimus is more hydrophobic than docetaxel hence requiring a greater lipid content. This comparison has led us to a relevant conclusion for liposomal formulation since it indicates that the lipid:drug ratio can be tuned depending on the nature of the encapsulated drug.

Unlike samples A, B, and C, in sample D, the amount of cholesterol was increased, reaching up to POPC: Chol of $7: 3$ ratio (Table 1). This was done to stabilize the structure of the lipid bilayer and increase the stability of the sample in biological serum for the intended application [24, 25]. However, it was found that $\% E E$ decreased to an average value of $44.13 \%$. This is due to the fact that cholesterol is positioned within the lipid layer and therefore obstructs the drug encapsulation by $50 \%$ since everolimus is also a hydrophobic molecule which is primarily positioned within the lipid bilayer [26] (Table 2).

Formulation E was performed with a lipid:drug 60:1 ratio and a 9:1 DOPC: Chol ratio, which showed lower encapsulation percentage than sample A which is composed of the same molar ratios (lipid:drug 60:1 and 9:1 POPC). However, the difference of sample $\mathrm{E}$ was that the type of PC used was DOPC instead of POPC as in A-D formulations. This was done to analyze the effect of double bonds (see Figure 5) in the entrapment efficiency of everolimus, as previously reported [12]. Therefore, a comparison of \%EE was made between liposomes composed of DOPC (with 2 


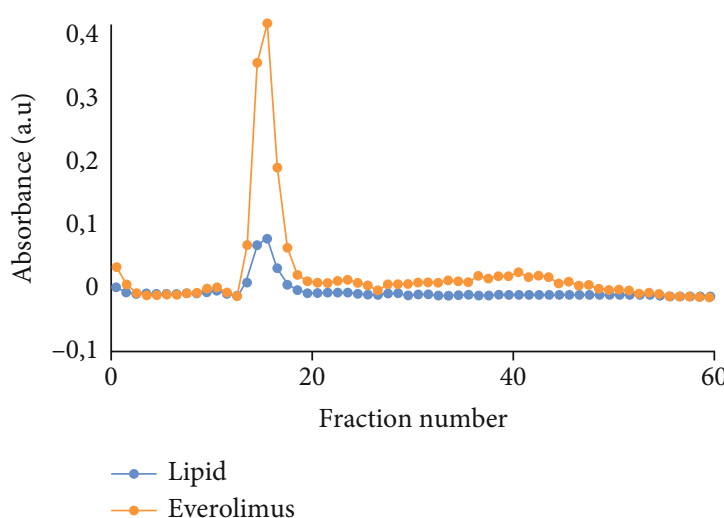

(a)

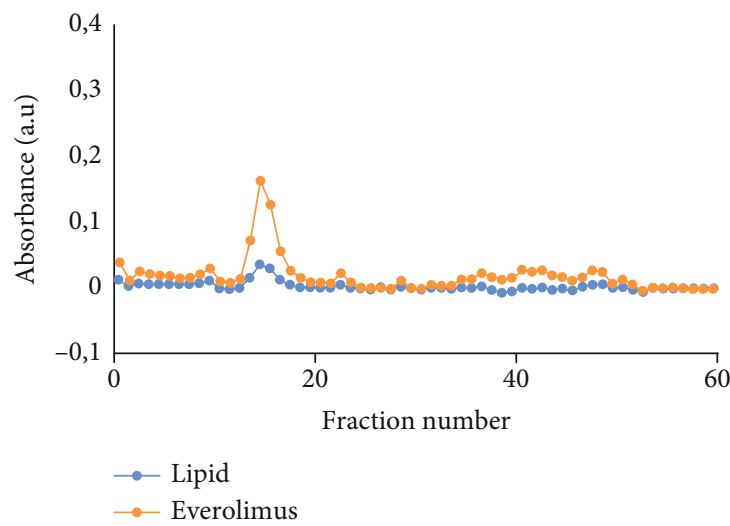

(c)

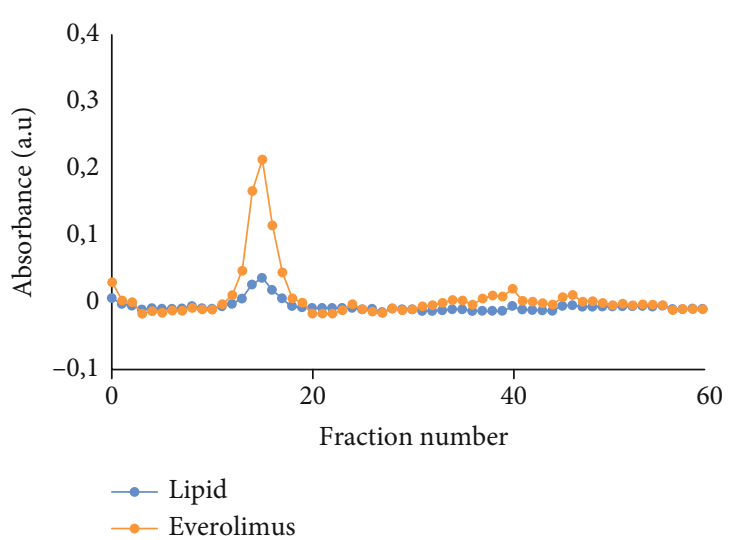

(b)

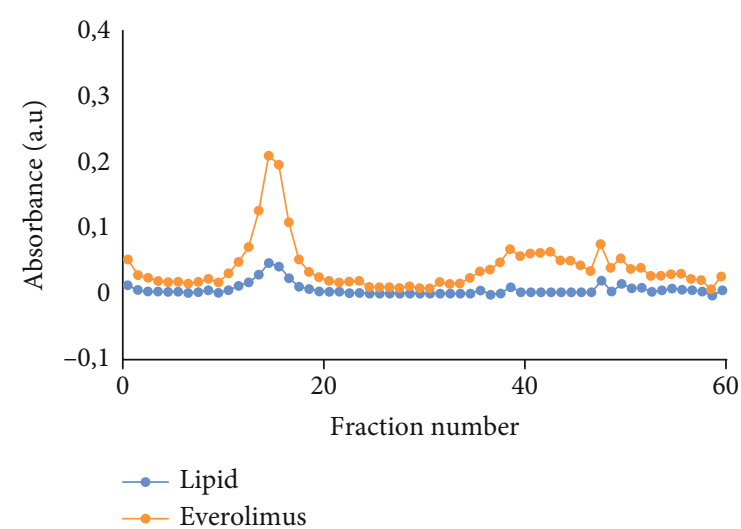

(d)

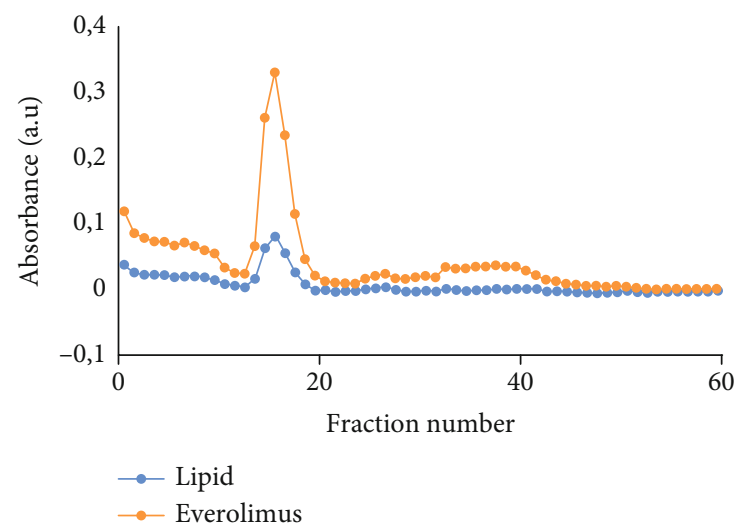

(e)

FIGURE 4: Entrapment analysis. UV-Vis extraction profile of samples (a), (b), (c), (d), and (e) obtained from the liposomes' purified fractions.

TABLE 2: Entrapment summary. Average \%EE obtained by UV-Vis at $278 \mathrm{~nm}$ of the samples (POPC: Chol: DOPC/lipid : drug).

\begin{tabular}{lcc}
\hline Sample & $\begin{array}{c}\text { Entrapment efficiency } \\
(\text { EE } \%)\end{array}$ & Standard deviation \\
\hline Blank & 11.51 & 6.07 \\
A $(9: 1: 0 / 60: 1)$ & 92.82 & 2.64 \\
B $(9: 1: 0 / 45: 1)$ & 45.27 & 0.30 \\
C $(9: 1: 0 / 30: 1)$ & 33.68 & 0.66 \\
D $(3: 7: 0 / 60: 1)$ & 48.69 & 5.76 \\
E $(0: 1: 9 / 60: 1)$ & 71.89 & 5.18 \\
\hline
\end{tabular}

double bonds) and POPC (with one double bond). As it can be observed in Table 2, the \%EE of sample E (with DOPC) was $20 \%$ below that in sample A. This observation is relevant to understand the entrapment of everolimus in liposomes since it demonstrates that everolimus interaction is not carried out primarily in the double bonds of PC as suggested by Iwase and Maitani [27]. In that study, the \%EE increases when formulation with DSPC: Chol $55: 45$ was used versus another sample composed of soy lecithin: Chol 95:5. This was attributed to the double bond of linoleic acid present in soy lecithin [12]. However, the formulation of DSPC: Chol $55: 45$ is estimated to have had a 14 -fold lower \%EE since 


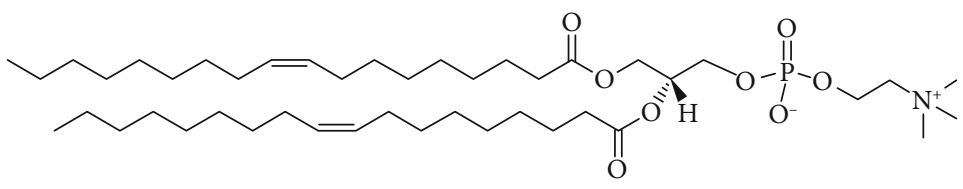

(a)

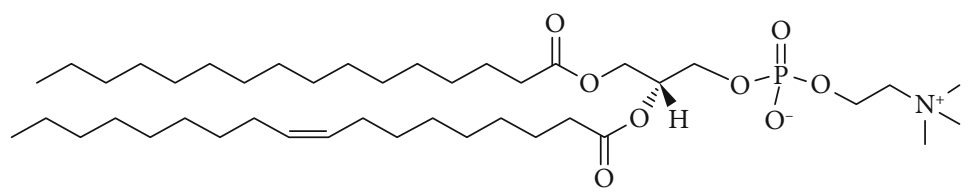

(b)

Figure 5: Chemical formula of (a) DOPC and (b) POPC.

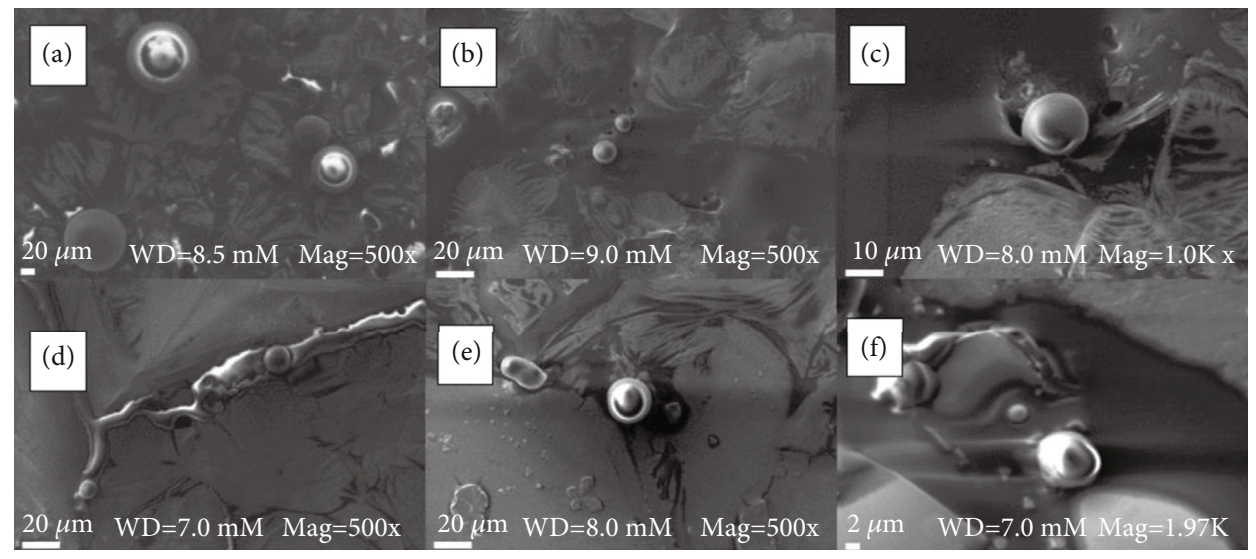

FIGURE 6: Morphological analysis. Images of liposomes obtained by scanning electron microscopy using an acceleration voltage of $5 \mathrm{kV}$ of (a) blank, (b) sample A, (c) sample B, (d) sample C, (e) sample D, and (f) sample E.

the percentage of cholesterol in the sample DSPC:Chol $55: 45$ used by Iwase et al. was 9 times higher than the sample composed of lecithin: Chol 95:5 [27].

3.4. Morphological Characterization of Liposomes. The morphology of liposomes was analyzed through SEM. All samples presented a spherical shape regardless of the molar ratios of the lipid and drug or if they were loaded with everolimus or the lipidic composition of liposomes. However, the sizes observed through SEM, shown in Figure 6, were significantly larger than those observed by DLS. We believe that this is due to the tendency of liposomes to fuse in the drying process during sample preparation, as seen in Figure 6. The observed change in morphology is due to the fact that water promotes the formation of liposomes, and, when evaporated, it can deform the original structure due to its high surface tension $[26,28]$. In addition, the increase in size affects the homogeneity of the sample since there is no control in size during the fusion process of liposomes. It is important to point out that the drying process can be affecting the size and shape of liposomes as well. In order to further understand the morphology and size of the loaded and preloaded liposomes, in future works, we will direct effort towards applying other techniques such as cryogenic TEM or environmental TEM, which could generate more in-depth information about morphology of liposomes and polydispersity index.

\section{Conclusions}

In the present work, it was determined how the lipid: drug and liposome lipid composition affect size, polydispersity index, stability, and encapsulation efficiency of the drugloaded liposomes. Specifically, we found that the formulation with the highest \%EE (95\%) was a liposome with a lipid: drug $60: 1$ ratio and with a lipid composition with POPCChol $9: 1$. It was also found that the $\% \mathrm{EE}$ of everolimus is directly proportional to the lipid:drug ratio and inversely proportional to the amount of cholesterol and double bonds present in PC. This finding could be relevant for the encapsulation of other hydrophobic drugs in liposomes following similar compositions. The obtained results suggest that the thin layer hydration and extrusion method represents a simple and reproducible method. This allowed to study the behavior of lipid-everolimus, thus permitting the tuning of encapsulation efficiency by modulating the lipid composition. As future work, morphological characterization could be done by an environmental SEM or cryogenic TEM in order to avoid liposomal fusion and to observe single liposomes and to compare the morphology between samples. The next research efforts 
will be focused to perform release kinetics analysis to determine the drug release profile and functionalize liposomes in polymers to further coat the metallic stents. The results reported in this work will be relevant for further applications of liposomes as drug delivery systems and for their use in coronary stents to prevent in-stent restenosis.

\author{
Abbreviations \\ PCI: Percutaneous coronary intervention \\ PC: $\quad$ Phosphatidylcholine \\ Chol: Cholesterol \\ HEPES: 4-(2-hydroxyethyl) piperazine-1-ethanesulfonic \\ acid \\ POPC: Palmitoyl oleyl phosphatidylcholine \\ DOPC: Dioleoyl phosphatidylcholine \\ MLV: Multilamellar vesicle \\ OFAT: One factor at a time \\ LUV: Large unilamellar vesicle \\ \%EE: $\quad$ Percentage of entrapment efficiency \\ PDI: Polydispersity index \\ L:F: $\quad$ Lipid to drug ratio.
}

\section{Data Availability}

The experimental data used to support the findings of this study are available from the corresponding author upon request.

\section{Conflicts of Interest}

The authors declare that there is no conflict of interest regarding the publication of this paper.

\section{Authors' Contributions}

G.T-F. contributed to the methodology, investigation, original draft preparation, review, and editing. A.G-H. contributed to the conceptualization, supervision, review, and editing. The founding acquisition, validation, review, and editing were done by Y.V-C. C.R. contributed to the resources. A.R-G. did the conceptualization, project administration, review, and editing. All the authors read and approved the final version prior to submission. Gabriela Torres-Flores and Azucena Gonzalez-Horta contributed equally to the work.

\section{Acknowledgments}

We are grateful to Dvorak Montiel-Condado and Abelardo Chávez-Montes who gently lent us their laboratory equipment. The technical help received from Elda Graciela Gómez, Felipe López, César Augusto García, Regina Elizabeth Vargas Mejía, and Daniel Mejia-Valdez is greatly appreciated. This work has been supported by the Advanced Manufacturing Focus Group of Tecnologico de Monterrey.

\section{Supplementary Materials}

The supplementary section is divided in two appendixes: appendix A, being tables with numerical data obtained for size, PDI, and zeta potential of liposomes and appendix B, being the statistical analysis numerical evidence of results stated in the manuscript. (Supplementary Materials)

\section{References}

[1] D. K. Arnett, R. S. Blumenthal, M. A. Albert et al., "2019 ACC/AHA guideline on the primary prevention of cardiovascular disease: a report of the American College of Cardiology/American Heart Association Task Force on Clinical Practice Guidelines," Journal of the American College of Cardiology, vol. 140, no. 11, pp. e596-e646, 2019.

[2] G. Oliva, M. Espallargues, and J. M. V. Pons, "Stents recubiertos de fármacos antiproliferativos : revisión sistemática del beneficio y estimación del impacto presupuestario," Revista Española de Cardiología, vol. 57, no. 7, pp. 617-628, 2004.

[3] N. A. Scott, "Restenosis following implantation of bare metal coronary stents: pathophysiology and pathways involved in the vascular response to injury," Advanced Drug Delivery Reviews, vol. 58, no. 3, pp. 358-376, 2006.

[4] A. S. Puranik, E. R. Dawson, and N. A. Peppas, "Recent advances in drug eluting stents," International Journal of Pharmaceutics, vol. 441, no. 1-2, pp. 665-679, 2013.

[5] K.-I. Joo, L. Xiao, S. Liu et al., "Crosslinked multilamellar liposomes for controlled delivery of anticancer drugs," Biomaterials, vol. 34, no. 12, pp. 3098-3109, 2013.

[6] S. Mallick and J. S. Choi, "Liposomes: versatile and biocompatible nanovesicles for efficient biomolecules delivery," Journal of Nanoscience and Nanotechnology, vol. 14, no. 1, pp. 755765, 2014.

[7] M. Danaei, M. Dehghankhold, S. Ataei et al., "Impact of particle size and polydispersity index on the clinical applications of lipidic nanocarrier systems," Pharmaceutics, vol. 10, no. 2, p. 57, 2018.

[8] V. Nele, M. N. Holme, U. Kauscher, M. R. Thomas, J. J. Doutch, and M. M. Stevens, "Effect of formulation method, lipid composition, and PEGylation on vesicle lamellarity: a small-angle neutron scattering study," Langmuir, vol. 35, no. 18, pp. 6064-6074, 2019.

[9] Q. Wu, K. S. Huang, M. Chen, and D. J. Huang, "Rapamycin enhances platelet aggregation induced by adenosine diphosphate in vitro," Platelets, vol. 20, no. 6, pp. 428-431, 2009.

[10] T. Palmerini, U. Benedetto, G. Biondi-Zoccai et al., "Longterm safety of drug-eluting and bare-metal Stents," Journal of the American College of Cardiology, vol. 65, no. 23, pp. 24962507, 2015.

[11] S. Granata, A. Dalla Gassa, A. Carraro et al., "Sirolimus and everolimus pathway: reviewing candidate genes influencing their intracellular effects," International Journal of Molecular Sciences, vol. 17, no. 5, p. 735, 2016.

[12] S. Pereira, R. Egbu, G. Jannati, and W. T. al-Jamal, "Docetaxelloaded liposomes: the effect of lipid composition and purification on drug encapsulation and in vitro toxicity," International Journal of Pharmaceutics, vol. 514, no. 1, pp. 150-159, 2016.

[13] T. B. Yu, Z. Shi, and S. H. Su, "Stable liposomal formulations of rapamycin and rapamycin derivatives for treating cancer," US Patent 2017/0065520A1, 2017. 
[14] A. Haeri, S. Sadeghian, S. Rabbani, M. S. Anvari, M. A. Boroumand, and S. Dadashzadeh, "Use of remote film loading methodology to entrap sirolimus into liposomes: preparation, characterization and in vivo efficacy for treatment of restenosis," International Journal of Pharmaceutics, vol. 414, no. 1-2, pp. 16-27, 2011.

[15] G. Rouser, A. N. Siakotos, and A. S. Fleisher, "Quantitative analysis of phospholipids by thin-layer chromatography and phosphorus analysis of spots," Lipids, vol. 1, pp. 85-86, 1966.

[16] A. Gonzalez-Horta, A. Matamoros-Acosta, A. ChavezMontes, R. Castro-Rios, and J. Lara-Arias, "Biodegradable nanoparticles loaded with tetrameric melittin: preparation and membrane disruption evaluation," General Physiology and Biophysics, vol. 36, no. 4, pp. 373-381, 2017.

[17] H. Martinez-Seara, T. Róg, M. Karttunen, I. Vattulainen, and R. Reigada, "Cholesterol induces specific spatial and orientational order in cholesterol/phospholipid membranes," PLoS One, vol. 5, no. 6, article e11162, 2010.

[18] H. Barman, M. Walch, S. Latinovic-Golic et al., "Cholesterol in negatively charged lipid bilayers modulates the effect of the antimicrobial protein granulysin," The Journal of Membrane Biology, vol. 212, no. 1, pp. 29-39, 2006.

[19] F. Giudice, Caracterización estructural de liposomas y su interacción con moléculas anfifílicas de interés farmacológico, Tesis Doctoral en Ciencias Químicas Biol Francesca Giudice Departamento de Química Biológica CIQUIBIC, 2016.

[20] J. Salcedo, A. Delgado, and F. González-Caballero, "The effect of $\mathrm{pH}$ on the electrical properties of the cholesterol/water interface. I. $\zeta$ potential," Journal of Colloid and Interface Science, vol. 133, no. 1, pp. 278-281, 1989.

[21] J. Qi, W. Zhang, X. Feng et al., "Thermal degradation of gelatin enhances its ability to bind aroma compounds: investigation of underlying mechanisms," Food Hydrocolloids, vol. 83, pp. 497510, 2018.

[22] A. Mirmohseni, J. Hosseini, M. Shojaei, and S. Davaran, "Design and evaluation of mixed self-assembled monolayers for a potential use in everolimus eluting coronary stents," Colloids and Surfaces B: Biointerfaces, vol. 112, pp. 330-336, 2013.

[23] M. Wu, L. Kleiner, F. W. Tang, S. Hossainy, M. C. Davies, and C. J. Roberts, "Surface characterization of poly(lactic acid)/everolimus and poly(ethylene vinyl alcohol)/everolimus stents," Drug Delivery, vol. 17, no. 6, pp. 376-384, 2010.

[24] D. C. Drummond, C. O. Noble, M. E. Hayes, J. W. Park, and D. B. Kirpotin, "Pharmacokinetics and in vivo drug release rates in liposomal nanocarrier development," Journal of Pharmaceutical Sciences, vol. 97, no. 11, pp. 4696-4740, 2008.

[25] C. Kirby and G. Gregoriadis, "The effect of the cholesterol content of small unilamellar liposomes on the fate of their lipid components," Life Sciences, vol. 27, no. 23, pp. 2223-2230, 1980.

[26] M. A. Rouf, I. Vural, J. M. Renoir, and A. A. Hincal, “Development and characterization of liposomal formulations for rapamycin delivery and investigation of their antiproliferative effect on MCF7 cells," Journal of Liposome Research, vol. 19, no. 4, pp. 322-331, 2009.
[27] Y. Iwase and Y. Maitani, "Preparation and in vivo evaluation of liposomal everolimus for lung carcinoma and thyroid carcinoma," Biological and Pharmaceutical Bulletin, vol. 35, no. 6, pp. 975-979, 2012.

[28] S. Park, R. A. Venditti, H. Jameel, and J. J. Pawlak, "Changes in pore size distribution during the drying of cellulose fibers as measured by differential scanning calorimetry," Carbohydrate Polymers, vol. 66, no. 1, pp. 97-103, 2006. 